\title{
RESPONSABILIDAD CIVIL EN LA CONSTRUCCIÓN DE VIVIENDAS \\ REFLEXIONES SOBRE LOS REGÍMENES LEGALES APLICABLES A LOS DAÑOS PROVOCADOS POR EL TERREMOTO DEL 27 DE FEBRERO DE 2010*
}

\author{
LIABILITY IN THE CONSTRUCTION OF BUILDINGS. \\ DISCUSSION ABOUT THE DIFFERENT LEGAL PATHS TO \\ RECOVER DAMAGES CAUSED BY CHILEAN'S EARTHQUAKE OF \\ FEBRARY 27, 2010.
}

\section{Hernán Corral TaLCIANi ${ }^{* *}$}

\begin{abstract}
RESUMEN: Tras el terremoto ocurrido en Chile el 27 de febrero de 2010, se ha suscitado una aguda controversia sobre si las empresas constructoras o inmobiliarias deben responder civilmente por los daños causados en las viviendas. El trabajo expone que frente a este problema no existe un único régimen legal, sino una diversidad de estatutos que conviven y que pueden ser utilizados por los afectados. Dos de ellos son de responsabilidad sin culpa, uno está en la Ley General de Urbanismo y Construcciones y otro en los artículos. 2003, 2004 y 2324 del Código Civil. A estos, debe agregarse el estatuto general de la responsabilidad por culpa contenido en el mismo Código. Se analizan los requisitos de estos estatutos, así como la posibilidad del afectado de optar por cualquiera de ellos. Se reflexiona sobre las defensas posibles de los demandados, en particular el caso fortuito, el cumplimiento de normas y la prescripción extintiva.
\end{abstract}

Palabras clave: responsabilidad civil, construcción, terremoto, caso fortuito, prescripción.

ABSTRACT: After February 27, 2010's earthquake in Chile, there have been much controversy on whether or not construction companies are liable of damages caused by collapse of their buildings. This work explores the different legal paths the victims can use to recover damages. Two of them are strict liability systems: the General Law of Urbanism and Constructions system of companies' liability, and articles 2003, 2004 and 2324 of the Chilean Civil Code. To these, the author adds the common system of liability by fault consecrated in Article 2314 of the Chilean Civil Code. The article provides analysis of the alternative causes of action offered to the victims and their requirements. It also discusses possible causes of exoneration by Defendants, in particular act of God, fulfillment of legal duties, and prescription.

Key words: Torts, Strict Liability, Construction, Earthquake, Act of God, Limitation Periods.

\section{DIVERSIDAD DE ESTATUTOS DE RESPONSABILIDAD APLICABLES}

Lo primero que debe constatarse en materia de reparación de daños producidos por un sismo como el del 27 de febrero de 2010 y sus réplicas en 
las construcciones dedicadas a uso habitacional, es que no existe un único régimen jurídico que resulte aplicable, sino una pluralidad de regímenes.

De partida deben considerarse las reglas del derecho común para la reclamación de indemnización de perjuicios derivados de un incumplimiento contractual y de un delito o cuasidelito civil, bajo el sistema subjetivo que rige en nuestro Derecho privado, cuyos factores de imputación son el dolo o la culpa.

Pero, superpuestos al derecho común, existen dos estatutos especiales sobre daños producidos por la construcción: el del Código Civil, integrado por los arts. 2003, 2004 y 2324, y el de la Ley General de Urbanismo y Construcciones (LGU) ${ }^{1}$, compuesto por sus arts. 18 y 19.

En estos estatutos especiales no tiene mayor relevancia si existe un vínculo contractual o no entre el demandante y demandado, por lo que no se producen diferencias importantes entre responsabilidad contractual 0 extracontractual.

Ambos estatutos son regímenes de responsabilidad objetiva, en el sentido de que eximen al demandante de la necesidad de probar dolo o culpa en la conducta del demandado ${ }^{2}$. Pero no se trata de una responsabilidad objetiva absoluta, fundada únicamente en la causación de un daño, sino de lo que la doctrina denomina modelos de responsabilidad estricta (expresión tomada del Derecho anglosajón: strict liability), calificada por factores de imputación adicionales a la causalidad pero que son diversos de la culpa subjetiva (actividad riesgosa, producto defectuoso, cosas peligrosas).

En el régimen especial del Código Civil la responsabilidad se deriva de que se haya verificado un "vicio", que puede ser de la construcción, del suelo o de los materiales.

En el régimen de la Ley de Urbanismo el factor de imputación es el concepto de "falla o defecto" de construcción.

En la actualidad la doctrina sostiene que los dos regímenes son compatibles, de modo que el perjudicado puede elegir entre uno u otro, aunque no mezclando disposiciones extraídas de ambos ${ }^{3}$.

Además, siempre queda reservada a la víctima la posibilidad de asilarse en el derecho común, que en materia extracontractual está contemplado en las reglas de los arts. 2314 y siguientes del Código Civil. La existencia de regímenes de responsabilidad estricta $u$ objetivada no impiden al afectado el demandar perjuicios (a veces superando así limitaciones establecidas en el régimen especial) si logra demostrar que el demandado causó el daño dolosa o culposamente ${ }^{4}$.

Dejamos fuera de este estudio otros estatutos que podrían aplicarse, como el de la responsabilidad contractual por vicios redhibitorios (arts. 1857 y ss. CC) o la responsabilidad de entes públicos, especialmente de las Municipalidades encargadas de conceder los permisos de edificación y de efectuar las recepciones de las obras (art. 142, Ley Orgánica Constitucional de Municipalidades, D. F. L. № 1,2006$)^{5}$.

\section{EL RÉGIMEN ESPECIAL DEL CÓDIGO CIVIL}


Para invocar el régimen del Código Civil es necesario que se pruebe que el edificio perezca o amenace ruina, en todo o en parte, por vicio de construcción, del suelo o de los materiales (art. 2003 regla $3^{a} \mathrm{CC}$ ) ${ }^{6}$.

No es necesario que el edificio se haya venido abajo, sino que basta la ruina potencial o inminente.

Algunos piensan que debe tratarse de una ruina física o material. Otros, entre los que me encuentro, siguiendo a la doctrina española, pensamos que también es ruinoso el edificio que, aunque no se haya destruido, resulte funcionalmente inutilizable para el fin para el que fue construido. Por ejemplo, si un edificio de departamentos no puede ser utilizado como vivienda. Se trataría de una ruina no material pero sí funcional ${ }^{7}$.

El demandante puede ser el dueño de la obra que contrató con el constructor (art. $2003 \mathrm{CC}$ ) o cualquier persona que haya sido perjudicada (art. 2324 CC). Por tanto, el adquirente posterior de la vivienda se encuentra legitimado para accionar de responsabilidad.

El demandado será el empresario constructor, sea persona natural o jurídica. También es legitimado pasivo el arquitecto o proyectista si el vicio es imputable a esta actividad profesional (art. 2004 CC).

La ruina, actual o potencial, del edificio debe haberse producido "en los cinco años subsiguientes a su entrega". Como veremos, la mayor parte de la doctrina se ha uniformado para entender que este plazo es un término de garantía, y no de prescripción extintiva de la acción de responsabilidad.

\section{EL ESTATUTO ESPECIAL DE LA LEY DE URBANISMO}

La Ley de Urbanismo atribuye responsabilidad sin culpa al "propietario primer vendedor" (art. 18 inc. $1^{\circ}$ LGU). Se trata de la constructora que vende o del empresario inmobiliario que manda construir para vender. El artículo 1.1.2 de la Ordenanza General de Urbanismo y Construcciones, define el propietario primer vendedor como el "titular del dominio del inmueble en que se ejecutó una obra y que realiza, a cualquier título, después de su recepción definitiva, la primera enajenación de la totalidad o de cada una de las unidades vendibles". Pero basta que se haya efectuado la construcción con este propósito, pues el propietario responde ante terceros aunque la construcción no haya sido transferida (art. 18 inc. 1ํㅡㄴ).

Se plantea el problema de las compraventas que a la fecha del sismo estaban ya otorgadas por escritura pública aunque aún no inscritas a nombre del comprador. Pensamos que si la tradición llega a efectuarse mediante la competente inscripción conservatoria, el adquirente podrá acogerse a este régimen ya que al momento de producirse el daño la empresa inmobiliaria tenía la calidad de "propietario primer vendedor"; en suma, basta que se haya perfeccionado la venta aunque el comprador no haya adquirido aún el dominio ${ }^{8}$.

La responsabilidad es amplia en cuanto a los daños: "todos los daños y perjuicios" (art. 18 inc. $1^{\circ} \mathrm{LGU}$ ). No hay duda por tanto que se indemniza el daño moral, que puede ser una partida importante en catástrofes tan 
traumáticas como un terremoto ${ }^{9}$.

Pero no es una responsabilidad objetiva absoluta. El demandante debe probar que los perjuicios provienen de "fallas o defectos" en la construcción, sea durante su ejecución "o después de terminada". En el caso de sismo las fallas o defectos pueden evidenciarse justamente con posterioridad a la terminación de la obra.

Además, la ley atribuye responsabilidad a los proyectistas por los errores en que hayan incurrido, si de ellos se han derivado perjuicios (art. 18 inc. $2^{\circ}$ LGU). Entendemos que al hablar de error aquí la ley vuelve al criterio de la culpa $^{10}$. Junto con los proyectistas, aunque subsidiariamente respecto del propietario primer vendedor, responde el revisor independiente (art. 116 bis inc. $\left.3^{\circ} \mathrm{LGU}\right)$.

Finalmente, es responsable el constructor por fallas, errores o defectos en la construcción, incluyendo las obras de los subcontratistas y el uso de materiales o insumos defectuosos (art. 18 inc $3^{\circ}$ LGU) ${ }^{11}$.

A los que se atribuye responsabilidad objetiva o sin culpa, propietario primer vendedor y constructor, se les admite ejercer acciones de reembolso contra el que por culpa produjo la causa del daño.

La ley extiende la responsabilidad de los sujetos que siendo personas jurídicas (sociedades) se han disuelto, a las personas naturales que eran representantes legales a la fecha de celebración del contrato (debe entenderse a la fecha de la primera venta) (art. 18 inc. 6ㄴ LGU).

Revisados los estatutos especiales de responsabilidad por defectos de construcción, podemos centrarnos en las causales por las cuales dicha responsabilidad podría ser desvirtuada. Son las posibles defensas disponibles para los demandados.

\section{LAS POSIBLES DEFENSAS}

\subsection{EL CASO FORTUITO}

Una de las defensas que pueden invocarse para exonerar de responsabilidad es el caso fortuito o fuerza mayor. Esta defensa procede en todos los regímenes mencionados, ya que el caso fortuito produce un quiebre del nexo de causalidad, de modo que los daños ya no son imputables al demandado sino al hecho que constituye la fuerza mayor o caso fortuito.

Como sabemos, el art. $45 \mathrm{CC}$, al definir el caso fortuito como el imprevisto a que no es posible resistir, pone como ejemplo justamente "un terremoto".

No obstante, los ejemplos que ofrece el artículo no son por sí mismos y en todo caso constitutivos de caso fortuito, porque deben cumplir con los requisitos de imprevisibilidad y de inevitabilidad ${ }^{12}$.

En el rubro de la construcción un temblor e incluso un terremoto no parece que constituyan por sí mismos hechos imprevisibles, al menos en un país como Chile que ha sido caracterizado como uno de los más sísmicos del planeta. De allí se explican las normas y criterios de construcción antisísmica que imperan en nuestra legislación urbanística. 
A pesar de los grandes sismos que han azotado al país, se encuentra poca jurisprudencia sobre el tema. Existe un antiguo caso que la Revista de Derecho y Jurisprudencia sintetiza con la siguiente doctrina: "Tratándose de un país como Chile en que son frecuentes los movimientos terrestres, no se puede decir que los temblores de mediana intensidad, sean un imprevisto que no sea posible resistir". Con las mismas palabras es reproducida desde Alessandri ${ }^{13}$ casi por todos los autores que tratan del tema, pero ella no está así contenida en el fallo, y en todo caso se trata de la sentencia de primera instancia, que fue luego confirmada por la Corte de Apelaciones de Santiago, y por fallo de reemplazo de la Corte Suprema. En el caso se trató de la muerte de un transeúnte ocasionada por la caída de un trozo de mampostería de un edificio ocurrido tres días después de un sismo, y en el que se demandó al propietario del edificio en virtud del art. 2323 CC. La sentencia aduce que "del informe evacuado por el señor Director del Observatorio Astronómico de Chile (...), se desprende que el fenómeno sísmico registrado en la noche del 17 de diciembre de 1937, fue de mediana intensidad, clasificado para Santiago en los números 5 y 6 de la escala sismológica de Rossi-Forel, o sea, de acuerdo con dicha tabla, fue un movimiento de tierra con incapacidad para producir perjuicios materiales" (cons. $9^{\circ}$ de la sentencia de primera instancia) ${ }^{14}$.

Más reciente es el fallo de la Corte de Apelaciones de lquique que, respecto del terremoto que afectó a la primera región el 13 de junio de 2005, juzgó que era constitutivo de caso fortuito para excusar la responsabilidad de la empresa de servicios sanitarios por la salida de agua que se produjo en el momento del terremoto, pero no para exonerar de los daños causados por la interrupción del servicio y otros perjuicios ocasionados por las réplicas ${ }^{15}$.

Puede verse que la discusión se centrará en si la magnitud, la intensidad y la eficacia destructiva del sismo de febrero de 2010, debe ser considerado imprevisible para agentes profesionales de la Construcción, lo que será diferente según el lugar en que estaba emplazado el edificio ${ }^{16}$.

Pero incluso aunque se llegue a la conclusión de que hubo caso fortuito, este no servirá para excluir la responsabilidad si además se comprueba que hubo fallas o defectos de construcción o que, de algún modo, se incurrió en negligencia. La doctrina ha señalado que en los casos en que confluyen en el hecho dañoso el caso fortuito y la culpa (y lo mismo debe decirse a otros factores de imputación equivalentes a la culpa como en el régimen de la Ley de Urbanismo), no cabe exoneración de la responsabilidad, aunque sí podría darse una disminución de ella ${ }^{17}$.

\subsection{LA AUSENCIA DE FALLAS O DEFECTOS DE CONSTRUCCIÓN}

Otra de las excusas que se puede intentar para excluir la responsabilidad estricta calificada es que no se verifica el factor de imputación que, tanto el régimen del Código Civil como de la Ley de Urbanismo, establecen para aplicar estos estatutos especiales de responsabilidad sin culpa: la existencia de vicios (Código Civil) o de fallas o defectos (Ley de Urbanismo) en la construcción.

Debe considerarse, sí, que la carga de la prueba del vicio, falla o defecto 
corresponde al demandante, aunque el colapso o destrucción de un edificio, sobre todo comparado con otros que, estando en situación similar, no sufrieron daños, podría ser indicio o elemento idóneo para construir presunciones judiciales de existencia de fallas o defectos de construcción. En este sentido, un fallo de la Corte Suprema ha sostenido que si la empresa inmobiliaria demandada contesta la demanda alegando que los defectos fueron reparados o que estos son de responsabilidad de los ocupantes del edificio, se produce una inversión de la carga de la prueba en contra de la demandada, la que deberá acreditar que no tiene obligación de reparar los daños, porque no existen las fallas o defectos imputados por la demandante ${ }^{18}$.

También será constitutivo de falla o defecto la comprobación de que en la obra no se cumplieron con las normas o criterios jurídicos aplicables a la construcción, siempre que ese incumplimiento pueda ser reputado causa de los daños producidos. Lo mismo sucederá si se comprueba que la constructora no cumplió con las especificaciones técnicas que fueron contratadas para la obra.

\subsection{EL CUMPLIMIENTO DE LAS NORMAS}

Muy ligado con lo anterior, una de las defensas que se puede emplear para evitar la responsabilidad y para negar que existe falla o defecto de construcción, es la de haberse ajustado la construcción a las normas existentes sobre construcciones antisísmicas.

En materia de responsabilidad de productos defectuosos, la directiva europea establece como excusa de responsabilidad del fabricante "que el defecto se debe a que el producto se ajusta a normas imperativas dictadas por los poderes públicos" (art. 7, letra e, Directiva 85/374, de 25 de julio de 1985). Como se ve, la excusa solo procede si el defecto proviene del cumplimiento de normas imperativas, es decir, que no hayan dejado la posibilidad de elaborar el producto de otra manera. No parece que existan este tipo de normas para la actividad de la construcción en lo referido a la prevención de daños por sismos. Las reglas aplicables son Normas Chilenas aprobadas por el Instituto Nacional de Normalización, que no son imperativas en el sentido indicado, sino que establecen bases o mínimos, desde los cuales son libres los constructores para aplicar mayores estándares de seguridad.

La Norma Chilena más importante en la materia es la NCh 433 of1998, Norma de Diseño Sísmico de Edificios. Pero también habrá que considerar la NCh 2369 of2003, Diseño Sísmico de Estructuras e Instalaciones Industriales; la NCh 2745 of2003, Análisis y Diseño de Edificios con Aislación Sísmica; la NCh 1508 of2008 Geotecnia - Estudio de Mecánica de Suelos; la NCh 430 of2008, Hormigón Armado. Requisitos de Diseño y Cálculo y NCh 428 of1957, Ejecución de estructuras de acero.

Debe tenerse en cuenta que las normas: NCh 433 of 1998, Norma de Diseño Sísmico de Edificios; NCh 2369 of2003, Diseño Sísmico de Estructuras e Instalaciones Industriales; NCh 430 of 2008, Hormigón Armado. Requisitos de Diseño y Cálculo y NCh 428 of1957, Ejecución de estructuras de acero, 
son mencionadas expresamente por el Decreto Supremo № 47, Vivienda y Urbanismo, de 1992, que contiene el texto de la Ordenanza de la Ley General de Urbanismo y Construcción. El art. 5.1.27 de la Ordenanza dispone que "El Revisor de Proyecto de Cálculo Estructural revisará el proyecto de acuerdo con las normas técnicas que se indican a continuación y verificará su cumplimiento en lo que le sea aplicable" y en la lista que le siguen aparecen las normas indicadas ${ }^{19}$. La NCH 433 es mencionada también por el art. 5.1.7 en relación con los planos de estructura.

Respecto de la naturaleza jurídica de estas regulaciones técnicas, el art. 5.5.7 de la Ordenanza dispone que "Las Normas Técnicas Oficiales que se citan expresamente en esta Ordenanza serán obligatorias en tanto no contradigan sus disposiciones". Se agrega que "La aplicación y cumplimiento de las Normas Técnicas Oficiales a que se alude en el inciso precedente, será de responsabilidad de los profesionales competentes y del propietario de la obra". Por esta remisión, las normas citadas, en especial la NCh 433 of. 1998 es equivalente a una norma reglamentaria, al remitirse a ellas un reglamento como es la Ordenanza de la Ley General de Urbanismo.

¿Es suficiente prueba de que no hay fallas o defectos constructivos la demostración de que los daños se produjeron aun cuando se cumplieron los requisitos mínimos que fijan estas normas?

Este problema se ha presentado en la doctrina chilena en relación con la culpa, y puede ser aplicable a otros factores de imputación de responsabilidad como el referido. Alessandri ha señalado que el solo hecho de que el demandado acredite que ha cumplido las normas de cuidado establecidas legalmente no excluye necesariamente la responsabilidad extracontractual 20. Lo mismo piensa Barros, aunque admite que "tratándose de actividades que están sujetas a extensivas regulaciones de base técnica, se puede asumir que la observancia de las normas legales y reglamentarias es al menos un indicio de diligencia"21. En este último sentido, el cumplimiento de las Normas chilenas referidas puede considerarse un indicio, aunque no una prueba definitiva y concluyente, de que los daños provocados por el sismo no se han debido a fallas o defectos constructivos.

\subsection{EL CUMPLIMIENTO DE LA LEX ARTIS}

Como en la responsabilidad médica, posiblemente el establecimiento de si hay vicios, fallas o defectos se deberá analizar en relación con los criterios de un correcto actuar en la profesión, que constituyen la lex artis. Según Barros, estos usos normativos pueden servir para determinar deberes de cuidado, y se expresan frecuentemente en buenas prácticas profesionales 0 empresariales $^{22}$. Con referencia a la responsabilidad por el terremoto del 27 de febrero de 2010, Emilio Sahurie sostiene que aunque las normas antisísmicas tengan por objeto evitar el colapso en sismos de intensidad excepcionalmente severa, entendido el colapso como destrucción o ruina en la estructura, "además, el examen de una eventual responsabilidad no puede ignorar los estándares que, al momento de la construcción, aconsejaban las buenas prácticas de construcción e ingeniería para zonas expuestas a 
movimientos telúricos mayores" 23 .

Pueden citarse como ejemplos de este tipo de reglas técnicas el Manual de Detallamiento para Elementos de Hormigón Armado preparado por el Instituto Chileno de Hormigón, que determina cómo debe quedar colocada la armadura después del hormigonado para obtener un buen comportamiento del edificio en caso de cargas estáticas o dinámicas (sísmicas) ${ }^{24}$.

\subsection{El costo EConómico alternativo}

También en materia de productos defectuosos, para saber si el diseño de un producto es defectuoso se suele utilizar el test del costo alternativo (riskutility test). Se valora la proporción entre el riesgo y los costos del diseño alternativo más seguro. Significa dimensionar la viabilidad económica de ofrecer el producto con las condiciones de seguridad que hubieran impedido el daño provocado.

En los casos de daños por defectos constructivos, habría que aclarar cuánto hubiera costado el construir el edificio para que no hubiera sufrido los daños por un sismo con las características del ocurrido, y qué viabilidad económica hubiera tenido ese producto a dicho costo.

También aquí se considerará la información que se brindó al adquirente de la vivienda sobre el carácter y la estabilidad de la construcción.

\subsection{LA FALTA DE COLAPSO TOTAL DE EDIFICIO Y LA EVITACIÓN DE PÉRDIDA DE VIDAS HUMANAS}

Se ha argüido como posible defensa que, tratándose de sismos de alta magnitud y poder destructivo, lo único que se puede pretender es que el edificio no se derrumbe sobre los moradores y así les dé tiempo para escapar con vida. Este sería el estándar de seguridad que podría esperarse y exigirse en sismos de alta intensidad como el ocurrido el 27 de febrero último.

Aunque es obvio que la prevención de la pérdida de vidas humanas es una expectativa mínima para una construcción segura, no puede descartarse que ese solo hecho demuestre que no haya fallas o defectos de construcción que impiden el goce de un bien que fue adquirido con el ánimo de mantenerlo por tiempo ilimitado y que muchas veces ha significado un esfuerzo patrimonial de envergadura en relación a la capacidad económica del comprador.

\section{EL PROBLEMA DE LA PRESCRIPCIÓN}

Después de la reforma de la ley № 20.016, de 2005, se dispusieron diversos plazos de prescripción en el régimen de la Ley General de Urbanismo:

a) Diez años para fallas o defectos que afecten la estructura soportante del inmueble, contados desde la recepción definitiva por la Dirección General de Obras Municipal. La Cámara Chilena de la Construcción ha dicho en publicación en los diarios y en su página web ${ }^{25}$ que "el concepto 
de "estructura soportante" no está definido en la ley, aunque se podría asimilar al de "elemento de estructura". En efecto, la Ordenanza define como "todo elemento resistente u orgánico de una construcción, como cimientos, muros soportantes, suelos, pilares, techumbres, torres y otros

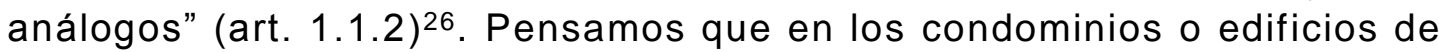
departamentos, no solo deben considerarse elementos de estructura aquellos que soportan la estructura completa del edificio o copropiedad inmobiliaria, sino también los de cada departamento o unidad.

b) Cinco años para fallas o defectos de los elementos constructivos o de instalaciones, contados desde la recepción definitiva por la Dirección General de Obras Municipal27.

c) Tres años para fallas o defectos que afecten elementos de terminaciones o acabado de las obras ${ }^{28}$, contados desde la inscripción en el Conservador de Bienes Raíces del dominio del inmueble a nombre del comprador.

Se entienden incluidas en cada grupo de fallas las que sean asimilables o equivalentes a ellos, y si no es asimilable o equivalente a ninguno, entonces rige un plazo de cinco años, que se contará desde la recepción definitiva.

A este respecto la declaración de la Cámara es confusa: "8) ¿Quién debe responder por los daños visibles no estructurales que se pudieran haber producido al interior de una vivienda por causa del terremoto? Los daños en elementos interiores -como tabiquería o azulejos, por citar algunos ejemplosdeberán ser repuestos por cada uno de los propietarios de los inmuebles afectados, quienes podrán o no contar con seguros para indemnizar daños derivados de riesgos catastróficos de la naturaleza, tales como terremotos, inundaciones, incendios a causa de terremotos u otros del mismo tipo. Estos seguros pueden estar incluidos en las pólizas de seguros que se toma con bancos e instituciones que otorgan créditos hipotecarios. En todo caso, la responsabilidad por este tipo de daños también podría recaer en el propietario primer vendedor, si los daños derivan de errores o fallas en la construcción" 29 . Como lo prueba esta frase final, la verdad es que no hay diferencia con los demás daños: los daños de tabiquerías o instalaciones son de responsabilidad del propietario primer vendedor o constructor cuando haya defectos o fallas en la construcción.

Pero si el perjudicado prefiere invocar el régimen especial del Código Civil, debe tenerse en consideración que la doctrina ha señalado que el plazo de cinco años desde la entrega es un plazo de garantía y no de prescripción ${ }^{30}$. Con ello, bastará que el daño por terremoto haya ocurrido dentro de ese término, para que nazca la acción de responsabilidad. Nacida así la acción, dentro de plazo de garantía, el perjudicado podrá ejercerla en el plazo de prescripción de cuatro años contados desde el momento en que se presentó el daño (art. $2332 \mathrm{CC})^{31}$. Esto puede llegar a extender el plazo hasta 9 años desde la entrega.

Si hay fallas estructurales esta extensión no tendrá mayor relevancia, ya que será más conveniente para el perjudicado recurrir al régimen de la Ley de 
Urbanismo, ya que esta le concede un plazo de diez años desde la recepción definitiva (que coincide normalmente con la entrega). Pero si hay fallas de construcción que, aunque no afecten la estructura, hagan inservible el inmueble para su fin natural podría alegarse el concepto de ruina funcional y allí el plazo del Código Civil resultaría mayor y más conveniente que el otorgado por el art. 18 de la Ley General de Urbanismo. También puede ser una opción más favorable para los adquirentes de viviendas en edificios a los que no se aplica el plazo de prescripción de diez años por ser anteriores a la entrada en vigencia de la ley que lo introdujo en el año 2005.

Aunque en general se entiende que la "entrega" a la que se refiere el Código Civil coincide con la recepción definitiva municipal ${ }^{32}$, podría considerarse que, en las construcciones sujetas al régimen de copropiedad inmobiliaria, el plazo se cuenta desde la entrega efectiva de la unidad o departamento al primer comprador ${ }^{33}$.

Por último, la víctima podría asilarse en las reglas generales de la responsabilidad y someterse a la carga de probar un delito o cuasidelito civil, es decir, que el daño fue causado por dolo o culpa de alguno de los agentes de la construcción. En tal caso, se aplicaría la regla de prescripción del art. 2332 CC que contempla un plazo de cuatro años desde la "perpetración del acto". La tendencia actual es interpretar el inicio del cómputo del plazo desde que se produce el daño y no desde que se consuma la conducta dolosa o negligente ${ }^{34}$. Existen pronunciamientos judiciales que acogen esta tesis para los daños en la construcción: así en el juicio de responsabilidad por los daños sufridos por el edificio Rey Sol II de Viña del Mar, como consecuencia del terremoto del 3 de marzo de 1985, la Corte Suprema estimó que, en atención a la regla del art. 2514 CC de que los plazos de prescripción extintiva se cuentan desde que la obligación se hizo exigible "tratándose de un ilícito como de autos, para que nazca el derecho a pedir indemnización, es necesario que se haya producido el daño. Antes no hay derecho para demandar perjuicios" 35 . Por su parte, la Corte de Apelaciones de Santiago en un juicio por daños de filtraciones de un edificio, sentó la tesis de que el plazo del art. 2332 debe computarse desde que se produce el perjuicio: "La jurisprudencia y la doctrina, en un principio, entendían que el plazo de prescripción de cuatro años comenzaba en el instante de la acción u omisión culpable o dolosa del autor del daño, aunque el daño se ocasionare posteriormente. La Excma. Corte Suprema sentó la buena doctrina, cambiando de opinión, y contó el plazo de prescripción desde el momento en que se produjo el daño, evitando con esta interpretación el absurdo de que la acción resulte prescrita antes de nacer, porque es requisito de la indemnización la existencia del daño que puede manifestarse con posterioridad al acto culposo o doloso" 36 .

\section{CUESTIONES DE EFECTOS DE LA LEY EN EL TIEMPO}

Es posible que en estos procesos se planteen problemas de efectos de la ley en el tiempo, ya que la Ley de Urbanismo ha sido objeto de dos reformas 
que constituyen hoy en día el estatuto que hemos analizado. La primera es la ley № 19.472, de 16 de septiembre de 1996, que estableció el régimen de responsabilidad objetivada o sin culpa centralizada en el propietario primer vendedor, pero con una prescripción de cinco años. Esta ley entró en vigencia noventa días después de su publicación en el Diario Oficial, esto es, el 17 de diciembre de 1996.

La segunda reforma fue efectuada por la ley № 20.016, de 27 de mayo de 2005, que entró en vigor también noventa días después de su publicación, esto es, el 25 de agosto de 2005. Por esta reforma se extendió el plazo a 10 años por las fallas en la estructura soportante.

Ambas leyes se hicieron cargo del conflicto de leyes en el tiempo y establecieron en un único artículo transitorio que sus modificaciones "solo se aplicarán a los permisos que se otorguen con posterioridad a su vigencia" (ley 19.472, artículo transitorio) o "solo se aplicarán a los permisos y autorizaciones que ingresen a tramitación con posterioridad a su entrada en vigencia" (ley 20.016, art. transitorio).

De esta forma, puede concluirse que el estatuto especial introducido por la ley 19.472 se aplica a todas las construcciones cuyos permisos de edificación fueron concedidos con posterioridad al 16 de diciembre de 1996. Pero los nuevos plazos de prescripción señalados en la ley 20.016 se aplican a las construcciones cuyos permisos 0 autorizaciones se presentaron (entraron a tramitación) después del 25 agosto de 2005.

Pero para las construcciones anteriores, que por el antiguo texto de la Ley de Urbanismo, rige el plazo de cinco años desde la recepción definitiva, podría el perjudicado optar por el régimen especial del Código Civil y en ese caso bastaría que el daño se hubiera producido dentro de los cinco años desde la entrega, para que comience un plazo de prescripción de cuatro años. También podría hacerse uso de las reglas comunes de responsabilidad y, sin importar la fecha de la entrega, correría un plazo de cuatro años desde el momento en que se produjo el daño, pero en este caso el demandante deberá probar el dolo o culpa del demandado.

\section{COMPETENCIA Y PROCEDIMIENTO.}

Si se ejerce la acción conforme al Código Civil son tribunales competentes los jueces ordinarios con jurisdicción civil. La competencia territorial la fijará el domicilio del demandado, no la situación del inmueble (art. 134 COT). El procedimiento aplicable es el juicio ordinario ${ }^{37}$.

Si se ejerce la acción del régimen especial de la Ley de Urbanismo también son competentes los jueces de letras en lo civil, pero su tramitación debe hacerse conforme al juicio sumario (art. 19 LGU). Existe la posibilidad de someter el proceso de arbitraje pero con árbitros de derecho o mixtos nombrados por la justicia.

No estimamos competentes a los jueces de policía local ni la aplicación del procedimiento de la Ley de Protección del Consumidor, ya que conforme al art. 2 letra e) esta ley se aplica a "Los contratos de venta de viviendas 
realizadas por empresas constructoras, inmobiliarias y por los Servicios de Vivienda y Urbanización, en lo que no diga relación con las normas sobre calidad contenidas en la ley $N^{\circ}$ 19.472"38. Aunque la ley 19.496 excluye expresamente solo el régimen de la Ley General de Urbanismo incorporado por la ley № 19.472, también deben considerarse inaplicable sus disposiciones cuando el perjudicado se acoja al régimen especial del Código Civil para mantener la consistencia en la jurisdicción: sería absurdo que el régimen de responsabilidad de la Ley General de Urbanismo se conociera por los jueces civiles en procedimiento ordinario mientras que la responsabilidad derivada del Código Civil fuera competencia de los jueces de policía local y con un procedimiento más simple y abreviado. Con ello no parece posible interponer acciones colectivas que solo se reconocen en la legislación de protección al consumidor (aunque limitadas al daño patrimonial).

Se ha planteado la posibilidad de perseguir la responsabilidad penal ya sea por la posible configuración de cuasidelitos de homicidio o lesiones, en caso de muertos o heridos por derrumbes, o de estafas a falta de este tipo de perjuicios personales. Si se ejerce la acción penal bajo el nuevo procedimiento penal debe recordarse que no puede accionarse civilmente contra terceros civilmente responsables, por lo que únicamente podría demandarse responsabilidad civil respecto de las personas naturales que sean consideradas imputables por el ilícito penal. Nada obsta, por cierto, para que se establezca primero el delito y luego se ejerza la acción civil contra los terceros responsables, en la medida en que no haya prescrito por haberse interrumpido civilmente.

\section{BIBLIOGRAFÍA CITADA}

AlEssandRI ROdRíguez, Arturo (1943/2005): De la responsabilidad extracontractual en el Derecho Civil chileno (Santiago, reimp. Editorial Jurídica de Chile) 559 pp.

BARROS BOURIE, Enrique (2006): Tratado de responsabilidad extracontractual (Santiago, Editorial Jurídica de Chile) $1230 \mathrm{pp}$.

CÁmARA CHILENA DE LA CONSTRUCCIÓN (2010): "10 claves sobre el terremoto y la calidad de las viviendas", 15 de marzo de 2010 Disponible en <http://www.cchc.cl/noticias/detalle.asp?id=4363>. [fecha de consulta: 19 de abril de 2010).

CoRRAL TALCIANI, Hernán (1996): "Daños causados por la ruina de edificios y responsabilidad civil del empresario y de los profesionales de la construcción", Revista de Derecho y Jurisprudencia t. 93, I, 1 pp. 39-70.

CORRAL TALCIANI, Hernán (1999): "La responsabilidad civil extracontractual en la construcción”, Gaceta Jurídica № 223 (1999) pp. 31-42.

CORRAL TALCIANI, Hernán (2003): Lecciones de responsabilidad civil extracontractual (Santiago, Editorial Jurídica de Chile) 423 pp.

COURT MurAsso, Eduardo (2008): "Algunas consideraciones en torno a la responsabilidad civil por vicios de la construcción en las legislaciones de España y Chile", en AA.VV., Estudios de Derecho Privado. Libro Homenaje 
al profesor Gonzalo Figueroa Yáñez (Santiago, Editorial Jurídica de Chile) pp. 275-308.

Figueroa Velasco, Patricio y Figueroa Valdés, Juan Eduardo (2006): Urbanismo y Construcción (Santiago, LexisNexis) 327 pp.

InStituto DEL Cemento y DEL HoRmigón DE ChILE: Manual de Detallamiento para Elementos de Hormigón Armado (2009) Disponible en <http://www.ich.cl/docs/libros/Manual_Detallamiento.pdf> [fecha de la consulta: 19 de abril de 2010).

LECAROS SÁNCHEZ, José Miguel (1998): "La responsabilidad civil en la actividad de la construcción", Revista de Derecho (Universidad Católica de Valparaíso), t. 19, pp. 295-307.

Rodríguez Grez, Pablo (2010): "Caso fortuito y responsabilidad civil”, El Mercurio, 20 de marzo de 2010, A-2.

SAHURIE, EMILIO, "Terremoto en Chile. Algunos aspectos en materia de seguros" (2010), Boletín Informativo Estudio Carvallo, 15, pp. 1-2.

URREJOLA SANTA MARía, Sergio (2007): La responsabilidad profesional de los agentes de la construcción (Santiago, LexisNexis) 114 pp.

\section{NORMAS CITADAS}

Código Civil.

Código Orgánico de Tribunales.

D. F. L. № 1, Ministerio de Interior, 9 de mayo de 2006, Fija texto refundido, coordinado y sistematizado de la ley № 18.695, Ley Orgánica Constitucional de Municipalidades. Diario Oficial 26 de julio de 2006.

Ley № 19.496, Establece Normas sobre Protección de los Derechos de los Consumidores. Diario Oficial, 7 de marzo de 1997.

D.L. № 1289, de 1975, Ley Orgánica de Municipalidades (derogada). Diario Oficial, 14 de enero de 1976.

Decreto con Fuerza de Ley № 458, Ministerio de Vivienda y Urbanismo, de 18 de diciembre de 1975, Ley General de Urbanismo y Construcciones. Diario Oficial, 13 de abril de 1976.

Ley № 19.472, Modifica el D.F.L. № 458, Ley General de Urbanismo y Construcciones, estableciendo Normas relativas a la Calidad de la Construcción. Diario Oficial, 16 de septiembre de 1996.

Ley № 20.016. Modifica Normas del Decreto con Fuerza de Ley № 458, de 1975, Ley General de Urbanismo y Construcciones, relativas a la calidad de la construcción. Diario Oficial, 27 de mayo de 2005.

Decreto Supremo № 45, Ministerio de Vivienda y Urbanismo, de 16 de abril de 1992, Fija Nuevo Texto de la Ordenanza de la Ley General de Urbanismo y Construcciones. Diario Oficial, 5 de junio de 1992.

\section{JURISPRUDENCIA CITADA}

Aldo Rojas Riobo con Aguas del Altiplano S.A. (2008): Corte de Apelaciones de lquique, 10 de marzo de 2008 (cas. en la forma), Base electrónica Legal Publishing № ID 38471. 
Comunidad Edificio Tikal con Pocuro Inmobiliaria S.A. (2004): Corte de Apelaciones de Santiago, 1 de septiembre de 2004 (apelación), Gaceta Jurídica № 291 (2004) p. 129.

El Quillay I con Inmobiliaria los Bosques S. A. (2009): Corte de Apelaciones de Santiago, 2 de diciembre de 2009 (apelación), Gaceta Jurídica № 354 (2009) p. 90.

Gustavo Rementería Rojas y otros con Terranorte S.A. (2009): Corte Suprema 21 de enero de 2009 (cas. en la forma), Base electrónica Legal Publishing № ID 41615.

Lara Catalán, Luis con llustre Municipalidad de Viña del Mar (2007): Corte Suprema 29 de octubre de 2007 (cas. en el fondo), rol № 2783-06, Base electrónica Microjuris MJJ16115.

Muñoz Valverde, Ricardo con Camus Camus, Manuel y otros (1995): Corte Suprema 18 de diciembre de 1995, Gaceta Jurídica № 186 (1995) p. 21.

Porzio v. de G. con Shell Mex Chile Ltda. (1941): Corte Suprema 26 de agosto de 1941 (cas. en el fondo), Revista de Derecho y Jurisprudencia, t. 39 (1941), II, sec. 1a, p. 203.

Wiedma W. Zenaida con Conde G., Román y otro (1967): Corte Suprema 1 de agosto de 1967 (cas fondo), Revista de Derecho y Jurisprudencia t. 64, II, sec. $1 \stackrel{a}{a}$, p. 265.

* El texto es elaboración de la charla ofrecida en el Auditorio del Colegio de Abogados de Chile, en un ciclo especial de conferencias relativas a la catástrofe del 27 de febrero de 2010. La charla fue realizada el día 17 de marzo del presente año.

** Licenciado en Ciencias Jurídicas, Pontificia Universidad Católica de Chile; Doctor en Derecho por la Universidad de Navarra, España. Profesor titular de Derecho Civil de la Facultad de Derecho de la Universidad de los Andes. Dirección postal: Facultad de Derecho, Universidad de los Andes, Av. San Carlos de Apoquindo 2.200, Las Condes, Santiago, Chile. Correo electrónico: hcorral@uandes.cl.

1 Esta ley está contenida en el Decreto con Fuerza de Ley № 458, Ministerio de Vivienda y Urbanismo, de 18 de diciembre de 1975, Ley General de Urbanismo y Construcciones. Diario Oficial, 13 de abril de 1976.

2 En contra, Court Murasso (2008) p. 304, quien sostiene que el régimen del Código Civil se basa en la culpa profesional, y que en el caso de ser responsabilidad extracontractual debe ser probada por el actor.

${ }^{3}$ Aunque en un primer estudio pensamos que debía reservarse la normativa del Código Civil para los casos de ruina: CORRAL TALCIANI (1996) p. 69; al analizar las normas introducidas por la ley № 19.472 en la Ley General de Urbanismo y Construcciones Ilegamos a la conclusión de que se trataba de regímenes opcionales: CORRAL TALCIANI (1999) p. 41. En el mismo sentido, se pronuncian LeCAROS SÁNCHEZ (1998) p. 296; URREJOLA SANTA MARía (2007) pp. 54-55; BARROS BOURIE (2006) pp. 772 y 775 nt. 309.

4 BARros Bourie (2006) p. 772, señala expresamente que, con independencia de los regímenes de responsabilidad estricta calificada del Código Civil o de la Ley General de Urbanismo, "por aplicación del derecho común de la responsabilidad civil, la responsabilidad extracontractual se dirige contra el responsable por dolo o negligencia de la ruina o defecto de construcción del edificio que provoca el daño".

5 Para imputar responsabilidad a la Municipalidad por los daños constructivos habrá que acreditar falta de servicio, para lo cual debe tenerse en cuenta el rol y las atribuciones que la ley entrega a la Municipalidad en la aprobación y supervisión de edificaciones. Actualmente el rol municipal es más bien de carácter formal y sus atribuciones son las de requerir que se adjunten los documentos exigidos legalmente y que estén firmados por los profesionales que se hacen responsable de su contenido. El art. 144 inc. 40 de la LGU dispone que el Director General de Obras debe revisar el cumplimiento de las normas 
urbanísticas aplicables a la obra, conforme al permiso otorgado. La Corte Suprema ha declarado que "entre las funciones de la Dirección de Obras no se encuentra la de verificar la calidad de las obras ejecutadas, sino que, por el contrario, su deber se vincula con el cumplimiento de normas técnicas de otra índole": Lara Catalán, Luis con llustre Municipalidad de Viña del Mar (2007): Corte Suprema 29 de octubre de 2007 (cas. en el fondo), rol № 2783-06, en Base electrónica Microjuris MJJ16115; en similar sentido, puede verse el caso Gustavo Rementería Rojas y otros con Terranorte S.A. (2009): Corte Suprema 21 de enero de 2009 (cas. en la forma), en: Base electrónica Legal Publishing № ID 41615. Sobre el rol de las municipalidades y su posible responsabilidad por falta de servicio, ver en este mismo sentido a BARros BourIE (2006) pp. 783-784.

6 Se exonera de responsabilidad el constructor al que el dueño de la obra le proporcionó los materiales siempre que el vicio sea de aquellos que el constructor no haya debido conocer en razón de su oficio (art. 2003 regla $3^{\underline{a}}$ en relación con el art. 2000 № 3 CC).

7 Corral Talciani (1996) p. 55;

Figueroa Velasco y Figueroa Valdés (2006) p. 252. En contra, Alessandri Rodríguez (reimp. 2005) pp. 306-307. Barros Bourie (2006) p. 775, nt. 309, dice que "no se podría sino compartir la conveniencia de esta interpretación extensiva del concepto de ruina, si en el derecho vigente la Ley de urbanismo y construcción no estableciera una regla de responsabilidad, que cubre las otras hipótesis de daño provocado por los constructores (...)", de lo cual deduce que es preferible mantener el concepto de ruina en un sentido estricto.

8 El supuesto plantea el problema de la aplicación de las reglas sobre riesgo de la cosa comprada contenidas en los arts. 1550 y $1820 \mathrm{CC}$, las que impondrían que el daño fuera soportado íntegramente por el comprador, a menos que el vendedor estuviera en mora de entregar. Pero debe recordarse que estas normas suponen que la pérdida o deterioro de la cosa vendida se haya producido por fuerza mayor o caso fortuito. Si se logra probar que el terremoto ha sido fuerza mayor o caso fortuito tampoco se podría aplicar el régimen de responsabilidad de la Ley de Urbanismo, ya que los daños no se deberán a fallas o defectos constructivos.

9 La jurisprudencia ha acogido la reparación del daño moral en la responsabilidad derivada de fallas de construcción: cfr. Gustavo Rementería Rojas y otros con Terranorte S.A. (2009): Corte Suprema 21 de enero de 2009 (cas. en la forma), en: Base electrónica Legal Publishing № ID 41615.

10 Corral TALCIANI (1999) p. 37.

11 La Ordenanza General de Urbanismo y Construcciones, Decreto Supremo № 47, Vivienda y Urbanismo, de 1992, agrega como sujeto responsable al inspector técnico de obra (ITO), disponiendo que "Ios inspectores técnicos serán subsidiariamente responsables con el constructor de la obra" (art. 1.2.8). Atendido el criterio de que la responsabilidad objetiva o estricta debe ser establecida por ley y no por reglamento, lo más seguro es concluir que este agente de la construcción responde por su negligencia profesional, que debe ser probada. Ello, sin perjuicio, de que la falta de cumplimiento por parte del inspector de alguna norma de la Ordenanza pudiera dar lugar a una "culpa contra la legalidad" si de ella se siguió el daño. La "subsidiariedad" a la que se refiere la norma parece referirse al propietario primer vendedor; es decir, el inspector es responsable en subsidio del propietario primer vendedor y conjuntamente con el constructor. El mismo criterio sostuvimos para la "responsabilidad subsidiaria" del revisor independiente a la que se refería el art. 116 bis de la Ley General de Urbanismo, antes de su modificación por la ley № 20.016: cfr. Corral TALCIANI (1999) p. 37.

12 Sahurie (2010) p. 1, sostiene que sucede con la mención del terremoto del art. 45 del Código Civil, algo similar al naufragio, también mencionado como ejemplo por dicho precepto, y para el cual los tribunales no lo aceptan per se como eximente de responsabilidad, sosteniendo desde muy antiguo que la responsabilidad del naviero dependerá de las negligencias que hubiese cometido el capitán o tripulación.

13 Alessandri Rodríguez (reimp. 2005) p. 436.

14 Porzio v. de G. con Shell Mex Chile Ltda. (1941): Corte Suprema 26 de agosto de 1941 (cas. en el fondo) en: Revista de Derecho y Jurisprudencia, t. 39 (1941), II, sec. 1a , p. 203. La escala de Rossi-Forel es la primera que midió la intensidad de los sismos según sus efectos, y fue sustituida por la de Mercalli, que es muy parecida. Tiene diez grados, el grado 5 , es descrito como un sismo que todos sienten y en que se mueven los muebles; el grado 6 es el que provoca el despertar de aquellos que duermen. Debe tenerse en cuenta que la escala de Richter es una escala de magnitud que mide la energía liberada por el movimiento 
telúrico (parte de números negativos y no tiene límite hacia arriba).

15 Aldo Rojas Riobo con Aguas del Altiplano S.A. (2008): Corte de Apelaciones de lquique, 10 de marzo de 2008 (cas. en la forma), en: Base electrónica Legal Publishing № ID 38471. La Corte señala que "la defensa del caso fortuito o fuerza mayor se encuentra demostrada desde que el 13 de junio de 2005, ocurrió un movimiento telúrico de considerable dimensión, provocando daños de toda índole, desastre que no requiere de prueba por ser de aquellos públicos, notorios y conocidos por la comunidad toda, de suerte que por la rotura de la válvula -motivo del primer escurrimiento de agua- no puede responder la parte demandada, resultando la petición infundada, conviniendo repetir, a mayor abundamiento, que la norma del Código Civil justamente menciona esta manifestación de la naturaleza como ejemplo de la defensa esgrimida..." (cons. $8^{\circ}$ ). Pero para la conducta posterior, la Corte estimó que no se cumplían los requisitos del caso fortuito: "al no haber adoptado la parte demandada, a través de sus agentes u operarios, todas las medidas tendientes a reparar la totalidad de los daños producidos y los eventuales, posibles de prever por tratarse este de un país que presenta permanente movimiento sísmico, con episodios severos desde que se tiene memoria, reveló desidia o negligencia en el cumplimiento del deber referido precedentemente, expresada en la falta de cuidado en el proceso de revisión de los estanques en la oportunidad en que repararon el primer defecto" (cons. $9^{\circ}$ ).

16 Debe constatarse que si se sigue la tesis de Pablo Rodríguez de medir la previsibilidad y la irresistibilidad del caso fortuito según el grado de diligencia de la culpa por la que debe responder el deudor, el caso fortuito se disuelve en la noción más amplia de ausencia de culpa. Pero esta asimilación entre caso y culpa no es aceptada por la doctrina chilena mayoritaria y, además, se observa difícil de aplicar en sede de responsabilidad extracontractual (donde la culpa no se gradúa) y más aún en modelos de responsabilidad objetivada o estricta. Con todo, en un artículo publicado en El Mercurio, el profesor Rodríguez insiste en que "frente a un 'caso fortuito' (como un terremoto) algunos obligados deben atajar sus efectos en tanto otros están exentos de responsabilidad a pesar del daño", según la medida del deber de diligencia y cuidado que le asigna la obligación. Llega a la conclusión de que quien construye un edificio y vende sus oficinas o departamentos responde de culpa leve por lo que el terremoto los exceptúa de responsabilidad si han respetado las normas técnicas relativas a la construcción o a la lex artis; cfr. Rodríguez GREZ (2010) p. 2.

17 Corral Talcianı (2003) p. 199, salvo si se prueba que el daño provocado por el caso fortuito se hubiera producido del mismo modo con independencia del comportamiento negligente del agente. En contra parece pronunciarse BARROS BOURIE (2006) p. 415, nt. 138, ya que sostiene que la reducción de la responsabilidad aceptada en su día por la jurisprudencia francesa, "debe mirarse con escepticismo, porque... lo que importa en el juicio de responsabilidad civil es si la negligencia fue significativamente determinante en el resultado, sin consideración de la intervención de otras causas".

18 Gustavo Rementería Rojas y otros con Terranorte S.A. (2009): Corte Suprema 21 de enero de 2009 (cas. en la forma), en: Base electrónica Legal Publishing № ID 41615.

19 Aunque debe tenerse en cuenta que la actuación del Revisor del Proyecto de Cálculo Estructural solo es aplicable a los edificios públicos (arts. 116 bis A LGU y 5.1 .26 de la OGU).

20 Alessandri Rodríguez (reimp. 2005) p. 132.

21 BARRos BoURIE (2006) p. 103.

22 BARROS BOURIE (2006) p. 104.

23 SAHURIE (2010) p. 2.

$24<$ www.ich.cl/docs/libros/Manual_Detallamiento.pdf> [Fecha de consulta: 17 de marzo de 2010].

$25<$ www.cchc.cl> [visitada el 17 de marzo de 2010].

26 También puede considerarse que la Ordenanza señala que la estructura soportante constituye uno de los criterios que deben tenerse en cuenta, junto con el material, para los efectos de distinguir las clases de construcción en la edificación (cfr. art. 5.3.1 OGU).

27 La Corte de Apelaciones de Santiago ha entendido que si han intervenido recepciones definitivas parciales que permiten la utilización de parte de la obra, el plazo de prescripción se cuenta desde la recepción parcial de la parte que fue afectada por el daño y no desde la última de las varias recepciones definitiva: cfr. El Quillay I con Inmobiliaria los Bosques S. A. (2009): Corte de Apelaciones de Santiago, 2 de diciembre de 2009 (apelación) en: Gaceta 
Jurídica № 354 (2009) p. 90.

28 No hay un concepto único de "terminaciones" en la Ordenanza, que utiliza el vocablo de diferentes maneras: cfr. arts. $4.1 .1 ; 4.37 ; 5.2 .6 ; 5.2 .8 ; 5.9 .5)$. En la historia de la tramitación de la ley se observa que se desistió de hacer una enumeración a título ejemplar de obras que se consideraban terminaciones (como cubiertas, hojalaterías, cielos, pisos, pinturas, puertas, ventanas, artefactos sanitarios o eléctrico) para evitar que la norma pudiera ser interpretada como restringida únicamente a los obras mencionadas.

29 <www.cchc.cl> [visitada el 20 de abril de 2010].

30 Así, Corral Talciani (1996) p. 66; Corral Talciani (1999) p. 41; Lecaros Sánchez (1998) p. 305; Urrejola Santa María (2007) p. 57; Figueroa Velasco y Figueroa Valdés (2006) p. 259; BARros Bourie (2006) pp. 785-786.

31 Si se trata del mandante que contrató con el constructor, algunos piensan que debe aplicarse el plazo de prescripción de las acciones contractuales ordinarias de cinco años: COURT MURASSO (2008) p. 307.

32 URREJola Santa María (2007) pp. 58-59.

33 Lo mismo podría aplicarse a las obras no acogidas a la ley de copropiedad porque lo habitual es que no coincida la recepción definitiva municipal con la entrega efectiva de la casa al comprador, que puede ser bastante posterior.

34 Barros Bourie (2006) pp. 922-924.

35 Muñoz Valverde, Ricardo con Camus Camus, Manuel y otros (1995): Corte Suprema 18 de diciembre de 1995, en: Gaceta Jurídica № 186 (1995) p. 21. La Corte se refería al plazo de prescripción de un año para hacer efectiva la responsabilidad de la Municipalidad, previsto en el art. 63 del D.L. № 1289, de 1975, antigua Ley Orgánica de Municipalidades. La Municipalidad demandada alegaba que la prescripción debía contarse desde el permiso o la recepción municipal del edificio, tesis que fue desestimada por la sentencia de casación.

36 Comunidad Edificio Tikal con Pocuro Inmobiliaria S.A. (2004): Corte de Apelaciones de Santiago, 1 de septiembre de 2004 (apelación), en: Gaceta Jurídica № 291 (2004) p. 129. La Corte parece hacer referencia al primer fallo de la Corte Suprema que sentó este criterio: Wiedma W. Zenaida con Conde G., Román y otro (1967): Corte Suprema 1 de agosto de 1967 (cas fondo) en: Revista de Derecho y Jurisprudencia t. 64, II, sec. 1a, p. 265. Acoge este criterio Barros BoUrie (2006) pp. 922-924, pero con la limitación de no extenderse a más de diez años (plazo de la prescripción extraordinaria) contados desde "que haya cesado la intervención causal".

37 UrRejola Santa María (2007) p. 58.

38 En este sentido también Figueroa Velasco y Figueroa Valdés (2006) pp. 270-271. 